\title{
Influência dos processos de secagem sobre o teor de flavonoides e na atividade antioxidante dos extratos de Baccharis articulata (Lam.) Pers., Asteraceae
}

\author{
Jackson Borgo, ${ }^{1}$ Carlos Augusto Gaiger Xavier, ${ }^{1}$ Dinara Jaqueline Moura, ${ }^{2}$ \\ Marc François Richter, ${ }^{1}$ Edna Sayuri Suyenaga ${ }^{* 3}$ \\ ${ }^{1}$ Universidade Luterana do Brasil, Programa de Pós-graduação em Genética e Toxicologia Aplicada. \\ Av. Farroupilha, 8001, Prédio 22, $5^{\circ}$ andar, 92420-280 Canoas-RS, Brasil \\ ${ }^{2}$ Universidade Federal do Rio Grande do Sul, Departamento de Biofísica, 210, Campus do Vale, \\ Av. Bento Gonçalves, 9500,Prédio 43422, 91509-900 Porto Alegre-RS, Brasil \\ ${ }^{3}$ FEEVALE Campus II, Instituto de Ciências da Saúde, RS 239, 2755, Prédio Lilás 201E, \\ 93352-000 Novo Hamburgo-RS, Brasil.
}

\begin{abstract}
RESUMO: O objetivo deste trabalho foi verificar o teor de quercetina obtido dos extratos de partes aéreas de Baccharis articulata (Lam.) Pers., Asteraceae, submetidas a diferentes técnicas de secagem, bem como a avaliação de sua atividade antioxidante in vitro. Foi verificada maior concentração deste flavonoide nas amostras secas em estufa, porém não houve diferença significativa na atividade farmacológica das amostras analisadas.
\end{abstract}

Unitermos: processos de secagem, quercetina, atividade antioxidante, in vitro.

\begin{abstract}
The Influence of drying processes on flavonoid level and the antioxidant activity of Baccharis articulata (Lam.) extracts". The objective of this work was to verify the influence of different drying processes on the levels of quercetin of the aerial parts of Baccharis articulata (Lam.) Pers., Asteraceae, as well as the evaluation of the in vitro antioxidant activity of these extracts. We demonstrated that the highest concentration of this flavonoid was detected in oven-dried samples, although there no significant difference in their pharmacological activity of all analyzed samples could be shown.
\end{abstract}

Keywords: drying process, quercetin, antioxidant activity, in vitro.

\section{INTRODUÇÃO}

Nos últimos anos têm-se observado um aumento significativo no consumo de plantas medicinais em nível mundial. Atualmente no Brasil, programas oficiais de saúde têm incentivado a exploração e/ou a produção sustentada de espécies medicinais, que vem sendo gradativamente implementados por muitos governos estaduais e municipais (Lourenzani et al., 2004).

Consequentemente a este crescente uso de plantas medicinais também são requeridos procedimentos de controle de qualidade pós-colheita, pois nem sempre estas espécies são produzidas sob condições apropriadas, o que poderá contribuir na qualidade do produto final (Corrêa et al., 2004).

A secagem de plantas medicinais é um processo crucial, a qual visa levá-las a baixos teores de umidade, o que permite a conservação adequada das mesmas, mantendo sua qualidade física e química por mais tempo (Radünz et al., 2002). Caso o teor de umidade residual estiver acima de $10 \%$ da base úmida nas drogas vegetais, pode favorecer o desenvolvimento de fungos e bactérias, bem como possibilitar a atividade hidrolítica de diversas enzimas presentes nas células vegetais, levando à reações de oxidação e degradação dos princípios ativos (Schulz, 2002), podendo desta forma, influenciar na sua atividade farmacológica. Sendo assim, é necessária a aplicação de metodologias de secagem apropriadas para cada espécie, visando assegurar os teores de substâncias ativas e, consequentemente, à saúde do consumidor (Corrêa et al., 2004).

Uma das espécies vegetais utilizadas na medicina popular gaúcha é Baccharis articulata (Lam.) Pers., Asteraceae, também conhecida por carquejinha, carquejadoce, carqueja-branca, vassoura ou carqueja-miúda (Lorenzi \& Matos, 2002). Trata-se de uma espécie pioneira, encontrada em grande número nos campos do Rio Grande do Sul (Simões et al., 1998).

Popularmente é empregada principalmente como digestivo, anti-séptico local e cicatrizante. Quanto a sua constituição química, são relatadas a presença de taninos, terpenos, saponinas, óleo volátil e flavonoides como 
luteolina, quercetina, santonina, absintina, acacetina, 7,4dimetil-apigenina, circimaritina, salvigenina, jaceidina, jaceosidina, além de circiliol, odoratina, platicarpanetina (Siqueira et al., 1988; Alonso, 1998; Simões et al., 1998; Agostini et al., 2005; Borella et al., 2006).

Mongelli et al. (1997) verificaram a atividade antioxidante do extrato aquoso obtido das partes aéreas de $B$. coridifolia DC., frente ao ensaio de determinação de substâncias reativas ao ácido tiobarbitúrico (TBARS), cujo $\mathrm{IC}_{50}$ foi igual a $556 \mu \mathrm{g} / \mathrm{mL}$. Estudando uma outra espécie, Tapia et al. (2004) observaram o efeito antioxidativo dos extratos de $B$. grisebachii, atividade conferida aos compostos fenólicos isolados $\left(\mathrm{IC}_{50}=10\right.$ $\mu \mathrm{g} / \mathrm{mL})$. Ambos estudos sugeriram que a presença destes metabólitos secundários poderiam justificar o uso popular destas espécies no tratamento de distúrbios gástricos. Pesquisas anteriores relataram que a formação de espécies reativas ao oxigênio (ERO), como o ânion superóxido e radicais hidroxila, poderiam exercer efeitos deletérios na membrana celular e tecidos, sendo que muitos problemas gastrintestinais estariam envolvidos com estresse oxidativo (Oh et al., 2001).

Sendo assim, este trabalho teve como finalidade investigar a influência de diferentes processos de secagem sobre o teor de quercetina de extratos obtidos das partes aéreas de $B$. articulata, bem como analisar a potencial atividade antioxidante in vitro das amostras submetidas a estas diferentes técnicas de estabilização.

\section{MATERIAL E MÉTODOS}

\section{Material vegetal}

Foram coletadas partes aéreas de Baccharis articulata (Lam.) Pers., Asteraceae, em início de setembro de 2005, em Novo Hamburgo. Confeccionouse uma exsicata, identificada pelo Dr. Sérgio Bordignon e depositada no Herbário do Museu de Ciências Naturais da ULBRA-Canoas, como HERULBRA-3547.

\section{Métodos de secagem}

Os métodos em que as partes aéreas de $B$. articulata foram submetidas para secagem foram: a temperatura ambiente $(\mathrm{N})$, estufa com circulação de ar forçado (E) e em forno de microondas (M). O delineamento experimental foi o totalmente casualizado com doze repetições, sendo cada repetição foi composta de $10 \mathrm{~g}$ de massa fresca das partes aéreas correspondente a cada tipo de secagem.

\section{Temperatura ambiente (N)}

Para a secagem em temperatura ambiente, as folhas foram expostas durante todo o tempo em local sombreado e ventilado, sem a presença de raios solares, cuja temperatura média manteve-se em $25^{\circ} \mathrm{C}\left( \pm 3{ }^{\circ} \mathrm{C}\right)$.

\section{Estufa com circulação de ar forçado (E)}

As folhas foram distribuídas uniformemente sobre prateleiras metálicas em uma estufa, marca Fanem modelo $315 \mathrm{SE}$, com a temperatura regulada a $40{ }^{\circ} \mathrm{C}( \pm 5$ $\left.{ }^{\circ} \mathrm{C}\right)$.

\section{Forno de microondas (M)}

O material vegetal foi colocado em um forno de microondas doméstico, de potência máxima de 900 watts (W), operando na frequência de 2450 Megahertz $(\mathrm{MHz})$. Um maço das folhas foi colocado por vez, sendo submetido a um minuto de aquecimento com potência máxima. Entre a submissão de um maço a outro, a porta do aparelho permaneceu aberta por um minuto, para eliminar a possibilidade de saturação do vapor d' água.

\section{Rendimento de biomassa seca}

Quando um material vegetal é submetido à secagem, ocorre uma redução considerável da sua massa seca em relação à da planta fresca. A faixa de redução de massa considerada aceitável, de acordo com Sartório (2000), está em torno de 20 a 75\%. Em todos os procedimentos, após uma perda significativa da umidade, as partes aéreas foram colocadas em dessecadores, para posterior pesagem das amostras. O material vegetal foi submetido aos métodos de secagem até obtenção de massa constante.

A perda de umidade de cada técnica foi avaliada de acordo com Anova seguida pelo teste Tukey, ao nível de $5 \%$ de probabilidade de erro.

\section{Preparo dos extratos}

Os extratos foram preparados segundo o doseamento de flavonoides totais, descrito na Farmacopéia Brasileira (2003). Após a obtenção dos extratos, foi retirada volumetricamente uma alíquota de $10 \mathrm{~mL}$ e colocado em funil de separação, juntamente com $10 \mathrm{~mL}$ de água e $10 \mathrm{~mL}$ de acetato de etila. Foi reservada a porção orgânica, sendo a fração aquosa novamente extraída com $6 \mathrm{~mL}$ de acetato de etila p.a. Este procedimento foi repetido duas vezes. A fração orgânica total foi submetida à extração adicionando $15 \mathrm{~mL}$ de água destilada. A solução final foi colocada em balão volumétrico de $25 \mathrm{~mL}$ e o volume foi completado com acetato de etila p.a. Este extrato foi submetido a uma corrente de nitrogênio até a completa secagem.

\section{Quantificação da quercetina}

Realizou-se a sua quantificação através de cromatografia líquida de alta eficiência (CLAE), utilizando cromatógrafo líquido Waters Alliance 2695, com detector Water UV/vis $2487(1=258 \mathrm{~nm})$, coluna de fase reversa 
Bondpack C 18 Waters $(300 \times 3,9-4 \mu \mathrm{m})$ e pré-coluna Bondpack C 18 Waters (10 x 3,9-4 $\mu \mathrm{m})$. A fase móvel foi constituída pelos sistemas A (água:ácido acético 95,5:4,5 v/v) e B (ACN 100\%), com vazão de $1,5 \mathrm{~mL} / \mathrm{mim}$.

As amostras N, E e M foram retomadas em 1 $\mathrm{mL}$ de acetonitrila (grau CLAE), colocando-as em balão volumétrico de $10 \mathrm{~mL}$ e completou-se o volume com água:acetonitrila (9:1 v/v) (Farmacopéia Brasileira, 2003). Em seguida, foram filtradas com filtros RC $0,45 \mu \mathrm{m}$ (Sartórious). Todos os solventes utilizados foram de alta pureza e filtrados em membrana GS em éster de celulose (0,22 $\mu \mathrm{m}$ de poro, $47 \mathrm{~mm}$ de diâmetro, Millipore) e desaerados em banho de ultra-som (Tecnal). O volume de injeção foi de $30 \mu \mathrm{L}$. A eluição foi controlada por software Waters. O sistema gradiente utilizado para as análises das amostras pode ser observado na Tabela 1 .

Tabela 1. Sistema de gradiente utilizado para as análises por CLAE.

\begin{tabular}{cccc}
\hline Tempo (min.) & Vazão (mL/min) & $\begin{array}{c}\text { Sistema A } \\
(\mathrm{mL})\end{array}$ & $\begin{array}{c}\text { Sistema } \\
\mathrm{B}(\mathrm{mL})\end{array}$ \\
\hline 0,0 & - & - & - \\
1,3 & 1,5 & 85 & 15 \\
5,0 & 1,5 & 40 & 60 \\
5,3 & 1,5 & 00 & 100 \\
7,3 & 1,5 & 00 & 100 \\
$7,6^{*}$ & 1,5 & 85 & 15 \\
$10,0^{*}$ & 1,5 & 85 & 15 \\
\hline
\end{tabular}

\section{Obtenção da curva-padrão de quercetina}

As curvas analíticas foram construídas, plotandose área do pico do composto versus concentração, utilizando-se cinco pontos da solução padrão nas faixas de concentrações. Preparou-se solução estoque de quercetina $(50 \mu \mathrm{g} / \mathrm{mL})$ e empregou-se o método do padrão externo com as concentrações de 0,$5 ; 1 ; 1,5 ; 2,0 ; 2,5 \mu \mathrm{g} /$ $\mathrm{mL}$. Alíquotas de $25 \mu \mathrm{L}$ foram filtradas e manualmente injetadas no sistema CLAE. Todas as análises foram realizadas em triplicata, em temperatura ambiente. A curva analítica foi obtida por regressão linear (área do pico versus concentração do padrão).

\section{Teste antioxidante in vitro-Reação enzimática a base de xantina oxidase}

A técnica empregada para a avaliação da atividade antioxidante foi descrita por Owen et al. (1996). A reação enzimática iniciou quando foram adicionados $4 \mu \mathrm{L}$ de xantina oxidase $(18 \mathrm{mU})$ em $1000 \mu \mathrm{L}$ da solução reagente em microtubos contendo extratos na faixa de concentração de 0,1 a $0,5 \mathrm{mg} / \mathrm{mL}$, ressuspendidos em solução tampão e incubados em termoagitador a $37^{\circ} \mathrm{C}$ por $3 \mathrm{~h}$. Para cessar a reação enzimática foi adicionado ácido clorídrico $(\mathrm{HCl})$ e o tubo colocado no gelo até análise dos ácidos diidróxibenzóicos (2,3-DHBA e 2,5-DHBA) via CLAE-DAD (cromatografia líquida de alta eficiência com detector photodiodearray). Paralelamente foram feitos controles negativos, sem a adição da enzima.

\section{Análise por CLAE-DAD}

Após a reação enzimática, as amostras foram centrifugadas a $1200 \mathrm{rpm}$ por $5 \mathrm{~min}$, e $20 \mu \mathrm{L}$ do sobrenadante foram injetados em cromatógrafo líquido, em condições cromatográficas descritas anteriormente por Owen et al., (2000), realizando-se a leitura em $325 \mathrm{~nm}$ para os ácidos diidróxi-benzóicos. Os resultados foram apresentados em porcentagem relativa na produção de ácidos diidróxibenzóicos, via reação radicalar, em comparação com a amostra sem tratamento, cujos dados foram avaliados de acordo com Anova (uma via) seguida pelo teste Tukey, ao nível de $1 \%$ de probabilidade de erro.

\section{RESULTADOS E DISCUSSÃO}

Em estudo realizado por Budel et al. (2004) verificou-se que o teor médio de umidade encontrado para Baccharis articulata (Lam.) Pers., Asteraceae, foi em torno de $8 \%$. No presente trabalho, verificou-se que houve perda significativa de umidade, em torno de $54 \%$ nas três técnicas empregadas, estando dentro da faixa de redução de massa úmida considerada aceitável. Observou-se também que não houve diferença significativa entre as massas constantes, obtidas entre os métodos de secagem $(p>0,05)$ (Tabela 2).

Segundo Corrêa et al. (2004) a técnica de secagem natural $(\mathrm{N})$ torna-se a mais viável para pequenos produtores, por evitar altos investimentos, bem como à menor possibilidade de alteração da composição química, como observada por Pinheiro (2002). Porém, trata-se ser um processo lento que pode facilitar a decomposição da droga vegetal em função da presença de enzimas e proliferação microbiana, favorecida muitas vezes pela alta umidade do ar atmosférico, como foi observado por Radünz et al. (2002) que demonstraram em estudos sobre a secagem do alecrim-pimenta, que este processo favoreceu o desenvolvimento de fungos, alteração da coloração do produto final e degradação do óleo volátil.

Em relação ao método de secagem $\mathrm{M}$, este requereu menor tempo que o primeiro, em torno de três minutos. Isto se deve, principalmente, ao fato das moléculas de água, presentes na droga vegetal, absorverem a energia microondas uniformemente, o que resulta numa rápida evaporação (Ohlsson, 1990). Muitos estudos relatam que o uso de microondas não tem afetado significativamente a qualidade dos nutrientes de alimentos, quando comparados ao aquecimento em forno convencional, como citado em pesquisa de Alajaji \& ElAdawy (2006) ao estudarem Cicer arietinum L. Peng et al. (1994) verificaram que a secagem não alterou o teor 
de nitrogênio em Oryza sativa L. Em pesquisas utilizando amostras de Phaseolus vulgaris L. e Zea mays L., Pastorini et al. (2002) demonstraram que este método não alterou os teores de carboidratos solúveis totais, açúcares redutores e aminoácidos. Porém, Lim \& Murtijaya (2007) observaram diminuição nos teores de compostos fenólicos totais, nos extratos obtidos pelas partes aéreas de Phyllanthus amarus (Schum. \& Thonn.) secas em microondas.

$\mathrm{O}$ método $\mathrm{E}$ apresentou vantagem em relação ao $\mathrm{N}$, por diminuir o tempo de secagem e ser um dos métodos mais empregados, atualmente, para a secagem de matérias-primas vegetais. Desde que, em condições adequadas de temperatura, geralmente não acima de $40^{\circ} \mathrm{C}$, mantém a qualidade da droga vegetal (Madhiyanon et al., 2007). A desvantagem desta técnica está na possibilidade de saturação da umidade, principalmente quando não há sistema de troca de ar. Devido à baixa condutividade termal dos vegetais durante o aquecimento, o calor é transferido para as suas partes internas, o que muitas vezes, não ocorre uma distribuição uniforme de energia térmica, conforme a natureza da droga vegetal (Feng \& Tang, 1998).

Dentre as técnicas de controle do processo extrativo comumente utilizadas, estão as técnicas cromatográficas, com as quais se torna possível, realizar avaliações tanto qualitativa quanto quantitativas. A análise da composição da droga vegetal e de suas preparações extrativas pode ser realizada através de cromatografia líquida de alta eficiência ou pelo emprego de cromatografia em camada delgada (CCD) (Mello, 1989).

Desta forma, no presente trabalho, foi caracterizada a presença de quercetina nos extratos obtidos das amostras secas pelos diferentes processos através da análise em CCD, frente à substância de referência. Obtevese curva analítica, por regressão linear Figura 1 (área do pico versus concentração do padrão), a qual apresentou boa linearidade, cujo coeficiente de correlação $\left(\mathrm{r}^{2}\right)$ da equação da reta foi igual a 0,9992 , possibilitando desta maneira a quantificação do analito pelo método do padrão do externo. Anteriormente ao doseamento, realizou-se a co-injeção das amostras junto à substância de referência quercetina, confirmando a sua caracterização. Os valores de concentração $(\mu \mathrm{g} / \mathrm{g})$ da quercetina presentes nas amostras estudadas foram calculados, como segue a Tabela 3.

Verificou-se maior concentração de quercetina nas amostras $\mathrm{E}$ e $\mathrm{M}$, que foram significativamente superiores a presente em N $(p<0,05)$. Sugere-se que a energia térmica envolvida nos processos $\mathrm{E}$ e $\mathrm{M}$, tenha permitido a ruptura de estruturas internas, que propiciaria maior extração compostos químicos, durante o processo de obtenção de extratos. Num estudo realizado por Albuquerque (2000) foi relatado que a secagem em estufa provocou o aumento de incidência de membranas de pontoações rompidas em Pinus taeda L., quando comparado ao de secagem a temperatura ambiente, que também apresentou rompimentos de membranas, porém de forma reduzida e localizada, o que poderia justificar o baixo rendimento observado nesta técnica. Roy et al. (2007) ao estudarem a influência do tratamento térmico sobre a atividade antioxidante de compostos fenólicos presentes em hortaliças, verificaram que o aquecimento a temperaturas inferiores a $50{ }^{\circ} \mathrm{C}$, preservou o conteúdo de compostos fenólicos e a atividade antioxidante, medida por meio da atividade sequestrante de radicais DPPH (difenilpicrilidrazila). Quanto à cocção, realizada em temperaturas usualmente mais altas, provocou efeitos negativos no conteúdo de compostos fenólicos, bem como na ação antioxidante. $\mathrm{O}$ mesmo fato foi observado durante a cocção em microondas por cinco minutos, em que se verificou a redução de compostos fenólicos totais.

Os flavonoides e outros compostos fenólicos presentes em plantas superiores são conhecidos por apresentarem potencial efeito antioxidante (Oh et al., 2001; Lima et al., 2006). Tapia et al. (2004) verificaram que os extratos aquosos de Baccharis grisebachii Hieron., inibiram a formação de radicais livres em diferentes sistemas in vitro em concentrações de $50-100 \mu \mathrm{g} / \mathrm{mL}$.

Dias et al. (2009) verificaram a atividade antioxidante de Baccharis trimera (Less) DC., em preparações da droga pulverizada, extrato bruto liofilizado, extrato bruto liofilizado da "resina" e frações clorofómica, acetato de etila, etanol absoluto e etanol $50 \%$, cujos dados apresentaram EC50 22,74 $\mu \mathrm{g} / \mathrm{mL}, 21,83 \mu \mathrm{g} / \mathrm{mL}$, $22,6 \mu \mathrm{g} / \mathrm{mL}, 24,93 \mu \mathrm{g} / \mathrm{mL}, 23,05 \mu \mathrm{g} / \mathrm{mL}, 17,18 \mu \mathrm{g} / \mathrm{mL}$, $18,19 \mu \mathrm{g} / \mathrm{mL}$, respectivamente. Num estudo anterior, Simões-Pires et al. (2005) observaram significativa atividade antioxidante dos extratos aquosos de B. trimera, B. crispa Spreng. e B. usterii Heering frente a DPPH, sugerindo tal ação à presença de ácidos cafeiolquínicos.

Segundo o ensaio da inibição da xantina oxidase, verificou-se potencial atividade antioxidante in vitro, em que a concentração de $100 \mu \mathrm{g} / \mathrm{mL}$ reduziu em cerca de $80 \%$ a produção de DHBA $(p<0,01)$ em relação ao controle negativo. Não foi observada diferença significativa $(p>$ $0,01)$ entre os três extratos, cujo $\mathrm{IC}_{50}(\mu \mathrm{g} / \mathrm{mL})$ de N, M e E foi de 82, 81 e 73, respectivamente (Figura 2). Os extratos obtidos pelas amostras E e M obtiveram maior concentração de quercetina, porém não foi verificada correlação dosedependente. Num estudo realizado por Chun-Mao Lin et al. (2002) foi determinado $\mathrm{IC}_{50}$ para a quercetina igual a $1,09 \mu \mathrm{g} / \mathrm{mL}$, para o mesmo ensaio antioxidante. Sugere-se que este efeito observado nos extratos N, E e M possa ser devido a presença deste flavonoide e/ou à ação sinérgica de outros compostos fenólicos, estáveis à ação térmica.

Estas observações estimulam a realização de estudos posteriores sobre a influência destas técnicas de secagem para a obtenção de extratos a serem analisados frente a ensaios farmacológicos, bem como estudos fitoquímicos. 
Tabela 2. Relação das massas entre os diferentes métodos de secagem.

\begin{tabular}{lcccc}
\hline $\begin{array}{l}\text { Método de } \\
\text { secagem }\end{array}$ & $\begin{array}{c}\text { Tempo } \\
\text { médio de } \\
\text { secagem }\end{array}$ & $\begin{array}{c}\text { Massa fresca } \\
(\mathrm{g})\end{array}$ & $\begin{array}{c}\text { Massa seca } \\
(\mathrm{g})\end{array}$ & $\begin{array}{c}\text { RMS } \\
(\%)\end{array}$ \\
\hline $\begin{array}{l}\text { Natural(N) } \\
\mathrm{n}=12\end{array}$ & 5 dias & $10,250 \mu 0,183$ & $4,917 \mu 0,138$ & 47,9 \\
amostras & & & & \\
$\begin{array}{l}\text { Estufa }(\mathrm{E}) \\
\mathrm{n}=12\end{array}$ & $20 \mathrm{~h}$ & $9,704 \mu 0,372$ & $4,390 \mu 0,131$ & 45,0 \\
$\begin{array}{l}\text { amostras } \\
\text { Microondas }\end{array}$ & & & & \\
$\begin{array}{l}\text { (M) } \mathrm{n}=12 \\
\text { amostras }\end{array}$ & 3 min & $10,124 \mu 0,172$ & $4,55 \mu 0,185$ & 44,9 \\
\hline
\end{tabular}

Tabela 3. Resultado da análise quantitativa de quercetina encontrada nos extratos obtidos das partes aéreas de $B$. articulata secas pelos métodos de N, E e M.

\begin{tabular}{lc}
\hline \multicolumn{1}{c}{ Tipo de secagem } & $\begin{array}{c}\text { Concentração na planta seca } \\
(\mu \mathrm{g} / \mathrm{g})\end{array}$ \\
\hline Natural $(\mathrm{N})$ & $167 \pm 3,4^{*}$ \\
Estufa $(\mathrm{E})$ & $334 \pm 6,5$ \\
Microondas $(\mathrm{M})$ & $300 \pm 5,8$ \\
\hline
\end{tabular}

Anova/Tukey: $p<0,05$ Média de leituras \pm epm (triplicata).

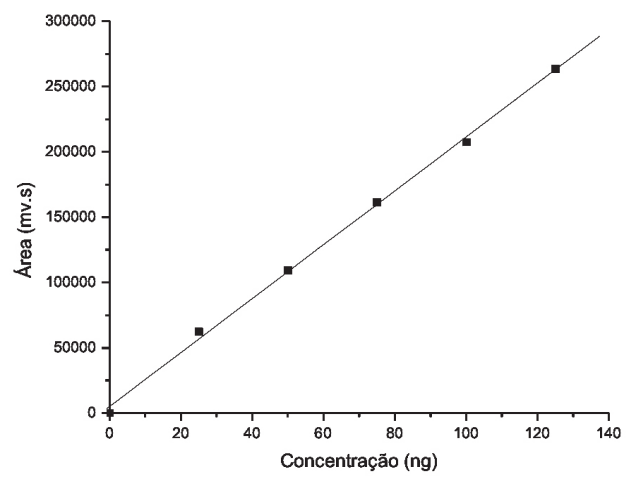

Figura 1. Representação gráfica de curva de quercetina obtida por CLAE.

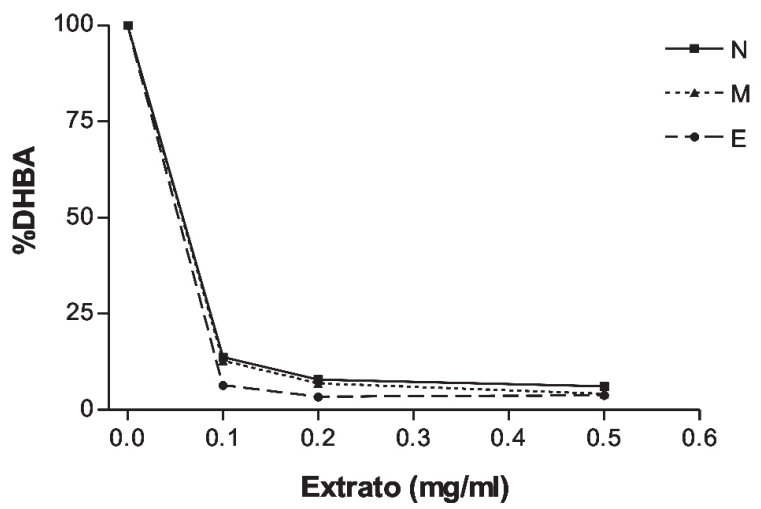

Figura 2. Efeito dos extratos obtidos pelas amostras N, E e M de B. articulata sobre a xantina oxidase.

\section{REFERÊNCIAS}

Alajaji SA, El-Adawy TA 2006. Nutritional composition of chickpea (Cicer arietinum L.) as affected by microwave cooking and other traditional cooking methods. J Food Comp Anal 19: 806-812.

Albuquerque CEC 2000. Efeito da secagem a $100{ }^{\circ} \mathrm{C}$ em membranas de pontoações de Pinus taeda L. Floresta Ambient 7: 129-136.

Alonso J 1998. Tratado de fitomedicina: bases clinicas $y$ farmacológicas. Buenos Aires: Isis.

Agostini F, Santos ACA, Rossato M, Pansera MR, Zattera F, Wasum R, Serafini LA 2005. Estudo do óleo essencial de algumas espécies do gênero Baccharis (Asteraceae) do sul do Brasil. Rev Bras Farmacogn 15: 215-220.

Borella JC, Duarte DP, Novaretti AAG, Menezes Jr A, França SC, Rufato CB, Santos PAS, Veneziani RCS, Lopes NP 2006. Variabilidade sazonal do teor de saponinas de Baccharis trimera (Less.) DC (carqueja) e isolamento de flavona. Rev Bras Farmacogn 16: 557-561.

Budel JM, Duarte MR, Santos CAM 2004. Parâmetros para análise de carqueja: comparação entre quatro espécies de Baccharis spp. (Asteraceae). Rev Bras Farmacogn 14: 41-48.

Corrêa RM, Corrêa SKVB, Pinto JEBP, Reis ES, Alves LA 2004. Rendimento de óleo essencial e caracterização organoléptica de folhas de assa-peixe submetidas a diferentes métodos de secagem. Cienc Agrotec 28: 339344.

Chun-Mao Lin, Chien-Tsu Chen, Hsiao-Hui Lee, Jen-Kun Lin 2002. Prevention of cellular ROS damage by isovitexin and related flavonoids. Planta Med 68: 365-367.

Dias LFT, Melo ES, Hernandes LS, Bacchi EM 2009 Atividades antiúlcera e antioxidante Baccharis trimera (Less) DC. (Asteraceae) Rev Bras Farmacogn 19: 309-314.

Farmacopéia Brasileira 2003. 4.ed. São Paulo: Atheneu.

Feng H, Tang J 1998. Microwave drying of diced apples in a spouted bed. J Food Sci 63: 679-683.

Lim YY, Murtijaya J 2007. Antioxidant properties of Phyllanthus amarus extracts as affected by different drying methods Food Sci Technol. 40: 1664-1669.

Lima AR, Barbosa VC, Santos Filho PR, Gouvêa CMCP 2006. Avaliação in vitro da atividade antioxidante do extrato hidroalcoólico de folhas de bardana. Rev Bras Farmacogn 16: 531-536.

Lorenzi H, Matos FJA 2002. Plantas Medicinais no BrasilNativas e Exóticas. Nova Odessa: Plantarum.

Lourenzani AEBS, Lourenzani WL, Batalha MO 2004. Barreiras e oportunidades na comercialização de plantas medicinais provenientes da agricultura familiar. Inform Econ 34: 1525.

Madhiyanon T, Adirekrut S, Sathitruangsak P, Soponronnarit S 2007. Integration of a rotary desiccant wheel into a hot-air drying system: Drying performance and product quality studies. Chem Eng Process 46: 282-290.

Mello JCP 1989. Desenvolvimento galênico de macerados de 
Baccharis trimera (LESS.) DC. Compositae (carqueja). Porto Alegre, 133 p. Dissertação de Mestrado, Programa de Pós-Graduação em Ciências Farmacêuticas, Universidade Federal do Rio Grande do Sul.

Mongelli E, Desmarchelier C, Rodriguez Talou J, Coussio J, Ciccia G 1997. In vitro antioxidant and cytotoxic activity of extracts of Baccharis coridifolia DC. $J$ Ethnopharmacol 58: 157-63.

Oh TY, Lee JS, Ahn BO, Cho H, Kim WB, Surch YJ, Cho SW, Hahm KB 2001. Oxidative damages are critical in pathogenesis of reflux esophagitis implication of antioxidants in its treatment. Free Radical Bio Med 30: 905-915.

Ohlsson T 1990. Microwave processing in the food industry. Eur Food Drink Rev 7: 7-11.

Owen RW, Wimonwatwatee T, Spiegelhalder B 1996. A high performance liquid chromatography system for quantification of hydroxyl radical formation by determination of dihydroxy benzoic acids. Eur J Cancer Prev 5: 233-240.

Owen RW, Spiegelhalder B, Bartsch H 2000. Generation of reactive oxygen species by the faecal matrix. GUT 46 : 225-232.

Pastorini LH, Bacarin MA, Abreu CM 2002. Secagem de material vegetal em forno de microondas para determinação de matéria seca e análises químicas. Cienc Agrotec 26: 1252-1258.

Peng S, Lasa MRC, Garcia FV, Cassman KG 1994. Microwaveoven drying of rice leaves for rapid determination of dry weight and nitrogen concentration. J Plant Nutr 17: 209217.

Pinheiro RC 2002. Abordagem fitoquímica, rendimento de óleo essencial de Lychonophora pinaster Mart. utilizando dois métodos de secagem. Lavras, 41 p. Dissertação de Mestrado, Pós Graduação em Agroquímica e Agrobioquímica, Universidade Federal de Lavras.

Radünz LL, Melo EC, Martins PM 2002. Secagem de alecrim pimenta (Lippia sidoides Cham.) em secador de leito fixo. Rev Bras Plant Med 5: 79-82.

Roy MK, Takenaka M, Isobe S, Tsushida T 2007. Antioxidant potential, anti-proliferative activities, and phenolic content in water-soluble fractions of some commonly consumed vegetables: effects of thermal treatment. Food Chem 103: 106-114.

Sartório ML 2000.Cultivo orgânico de plantas medicinais. Viçosa: Aprenda Fácil.

Schultz V 2002. Fitoterapia racional: um guia de fitoterapia para ciências da saúde. Barueri: Manole.

Simões CMO, Mentz LA, Schenckel EP, Irgang BE, Stchmann JR 1998. Plantas da medicina popular do Rio Grande do Sul. Porto Alegre: UFRGS.

Simões-Pires CA, Queiroz EF, Henriques AT, Hostettmann K 2005. Isolation and on-line identification of antioxidant compounds from three Baccharis species by HPLC-UVMS/MS with post-column derivatisation. Phytochem Analysis 16: 307-314.
Siqueira NCS, Alice CB, Thiesen FV 1988. Aspectos farmacognósticos e perfil cromatográfico dos constituintes de Baccharis articulata (Lam.) Pers. Compositae. Cad Farm 4: 63-76.

Tapia A, Rodriguez J, Theodulo C, Lopez S, Feresin GE, Schmeda-Hirschmann G 2004. Free radical scavengers and antioxidants from Baccharis grisebachii. $J$ Ethnopharmacol 95: 155-161. 